Supporting Information

\title{
Synthesis of Carbon Nanotubes by Rolling Up Patterned Graphene Nanoribbons Using Selective Atomic Adsorption
}

\author{
Decai Yu and Feng Liu ${ }^{*}$ \\ Department of Materials Science and Engineering, University of Utah, Salt Lake City, \\ UT 84112
}

\section{First-Principles Molecular Dynamics}

The first-principles MD simulations were performed using the pseudopotential plane-wave method within the local density approximation as implemented in the VASP code. ${ }^{1}$ We used single $\Gamma$ k-point for Brillouin zone sampling and plane wave cutoffs of 21.3 and 31.2 Ryd for H-C and F-C systems, respectively. The super cell dimension is $27.016 \times 4.2532 \times 23 \AA^{3}$ with a vacuum layer of $\sim 15 \AA$. The system contains a maximum of about 90 atoms (Fig. 1) with the graphite layer underneath the GNR fixed at the bulk position. The equation of atomic motions is integrated using the Verlet algorithm with a time step of 0.5 fs. The total simulation time is up to $3 \mathrm{ps}$.

\section{Classical Molecular Dynamics}

The classical MD simulations were performed using a modified form of the bondorder potential by Brenner et al.. ${ }^{2}$ A new bond-order term was introduced for $\mathrm{C}-\mathrm{H}$ bond to give the $\mathrm{H}$ adsorption energy $(\sim 0.9 \mathrm{eV})$ on graphite as predicted by the first-principles calculation, while the original Brenner potential predicted a $\mathrm{H}$ adsorption energy about $\sim 1.7 \mathrm{eV}$ higher. We also developed our own Lennard-Jones potential parameters to give a $\sim 42 \mathrm{meV} /$ atom interlayer cohesive energy of graphite, in accordance with the 
experiments. ${ }^{3}$ Relative to the first-principles calculations, we used a larger system with two graphite layers underneath the GNR and fixed the bottom layer at the bulk position. The system contains $\sim 1200$ atoms (Figs. 2 and 4). The periodic boundary condition along the ribbon length (i.e., tube axis) direction represents an infinite long tube, as the tube length is usually much larger than its diameter. As a cautionary check, we have also carried out simulations with finite ribbon length and free ends, and the results remain the same. The equation of atomic motions is integrated using the Verlet algorithm with a time step of $0.2 \mathrm{fs}$. The total simulation time is up to $2 \mathrm{~ns}$.

\section{Monte Carlo Algorithm}

A Metropolis Monte Carlo algorithm was used to simulate deposition of $\mathrm{H}$ atoms onto the GNR surface. The $\mathrm{H}$ atoms are randomly deposited on top of any carbon atoms (including edge atoms) that have not adsorbed $\mathrm{H}$ atoms. The probability for the adsorption depends on the local $\mathrm{H}$ coverage of the surface, which guarantees a roughly uniform $\mathrm{H}$ coverage. The rate of deposition, faster than the experimental rate, ${ }^{4}$ was chosen to make sure the system is well equilibrated before each new $\mathrm{H}$ atom is adsorbed. 


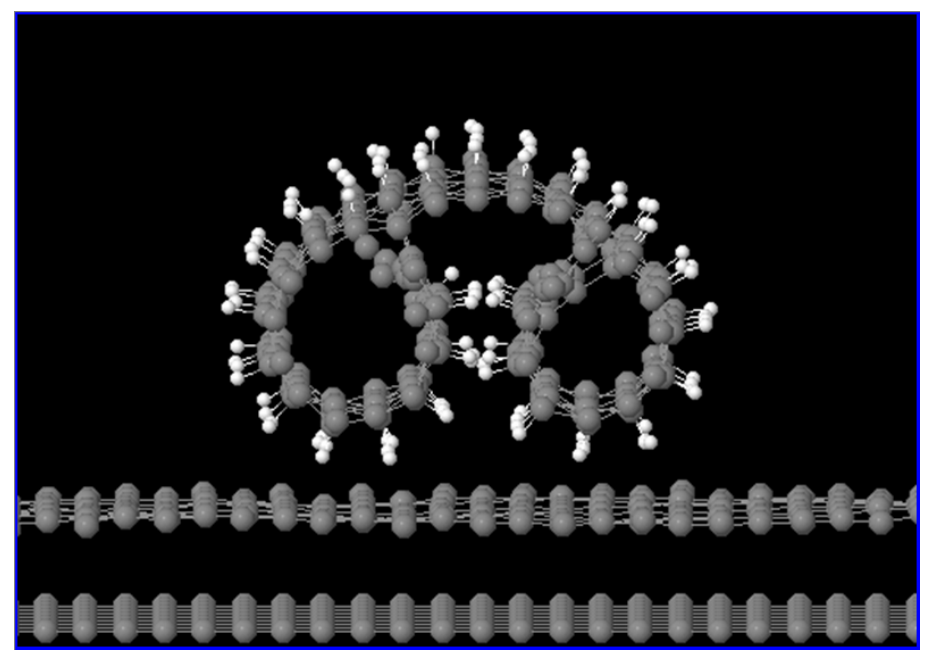

Supplementary Figure 1: The formation of double tubes. This happens when the patterned GNR width is much larger than the predicted circumference of the SWNT driven by a certain magnitude of stress. White and gray balls represent $\mathrm{H}$ and $\mathrm{C}$ atoms, respectively.

\section{Captions for Supplementary Online Videos \\ Supplementary video 1:}

First-principles MD simulation showing CNT formation driven by $\mathrm{H}$ adsorption ( $50 \%$ coverage) induced stress on patterned GNR. White and turquoise balls represent $\mathrm{H}$ and $\mathrm{C}$ atoms, respectively. (MPEG; 1.3 MB)

\section{Supplementary video 2:}

First-principles MD simulation showing CNT formation driven by $\mathrm{F}$ adsorption ( $45 \%$ coverage) induced stress on patterned GNR. Green and turquoise balls represent fluorine and carbon atoms, respectively. (MPEG; 1.3 MB)

\section{Supplementary video 3:}


Classical MD simulation of CNT formation driven by $\mathrm{H}$ adsorption (45\% coverage) induced stress on single-layer patterned GNR. Atom label is the same as in Video 1. The video shows two stages of the simulation process: (1) $300 \mathrm{~K}$ tube formation and (2) 1800K hydrogen desorption. (MPEG; $2.9 \mathrm{MB}$ )

\section{Supplementary video 4:}

Classical MD simulation of CNT formation driven by $\mathrm{H}$ adsorption $(50 \%$ coverage) induced stress on single-layer patterned GNR. Atom label is the same as in

Video 1. The video shows two stages of the simulation process: (1) $300 \mathrm{~K} \mathrm{H}$ adsorption and tube formation and (2) 1800K hydrogen desorption. (MPEG; 2.5MB)

\section{Supplementary video 5:}

Classical MD simulation of CNT formation driven by $\mathrm{H}$ adsorption $(50 \%$ coverage) induced stress on double-layer patterned GNR. Atom label is the same as in Video 1. The video shows two stages of the simulation process: (1) $300 \mathrm{~K}$ tube formation and (2) 1800K hydrogen desorption. (MPEG; 2.6MB)

(1) Kresse, G.; Hafner, J. Phys. Rev. B 1993, 47, 558-561.

(2) Brenner, D. W.; Shenderova, O. A.; Harrison, J. A.; Stuart, S. J.; Ni, B.; Sinnott, S. B. J. Phys.-Cond. Matt. 2002, 14, 783-802.

(3) Girifalco, L. A.; Lad, R. A. J. Chem. Phys. 1956, 25, 693-697.

(4) Ruffieux, P.; Groning, O.; Bielmann, M.; Mauron, P.; Schlapbach, L.; Groning, P. Phys. Rev. B 2002, 66, Art No. 245416. 